\title{
Based on the Quickbird remote-sensing image of Aoshan vegetation extraction
}

\author{
Li xue-xian, Wang guang-wei,Pan hong-jun,Qi chang-song \\ Department of Computer,Zhejiang Ocean Uiversity \\ Zhejiang, Zhoushan, China \\ Correspondingauthor :Wang guang-wei(1977-), Department of Computer, Zhejiang Ocean Uiversity, \\ Email:wangguangweitx@163.com
}

\begin{abstract}
On the analysis of terrain spectral features of Aoshan remote-sensing image, we identify spectral differences between the vegetation and other surface features, it combines mathematical morphology with vegetation distribution planning to complete the vegetation area extraction. Thus it can effectively realize the supervision of the regional vegetation.
\end{abstract}

Keywords-remote-sensing image; mathematical morphology; vegetation extraction

\section{INTRODUCTION}

The vegetation is the most active and valuable of the elements and indicating factor in global change. Vegetation affects the energy balance of the earth-atmosphere system, it plays an important role in the climate, hydrological and biochemical cycles. Vegetation is a sensitive indicator of the environmental impact for climatic and human factors. With the development of China's remote sensing images, the use of remote sensing data for vegetation information has been widespread. Aoshan is located in Zhoushan City, Zhejiang Province, it has a very rich natural resources and surrounded by the sea and surface coverings complex. Due to the complexity of the Aoshan specific terrain type distribution and the wide range of other factors, the accurate extraction of Aoshan will have a certain degree of difficulty and significance.

In this paper the source of data is the multispectral quick bird remote sensing image of Aoshan, we use the IDL language, and through the remote sensing image of vegetation index model and the use of mathematical morphology, vegetation area of Aoshan accurately extracted from other features.

\section{VEGETATION INDEX EXTRACTING}

Remote sensing image, it recording on the surface object to the electromagnetic wave reflection information and its own outward radiation information, in the near infrared, infrared and short wave infrared part, almost all the incident energy was absorbed by water. Therefore, the reflectance of water is particularly low. While soil, vegetation, buildings, etc in these bands absorb less energy, they have a high reflectance, so that the water body with them have a clear distinction $^{[1]}$. So using this difference, the vegetation can be separated from other features.

Vegetation index as a simple and effective reference, it has a high value in terms of vegetation monitoring.Vegetation index is a index of using satellite detection data of linear or nonlinear combination to reflect the existence, quantity, quality, condition, time and space distribution features of vegetation. Normalized differential vegetation index(NDVI) ${ }^{[2-3]}$ is a technology of normalized ratio index based on infrared and near-infrared bands. The formula of Normalized differential vegetation index shows in formula(1):

$N D V I=(p(N I R)-p(R E D)) /(p(N I R)+p(R E D))$

Where: NIR-near infrared, RED-infrared.

Following the Normalized differential vegetation index model calculation, the vegetation can separate form other surface features. Then we will get the NDVI vegetation index figure, it shows in figure 1.

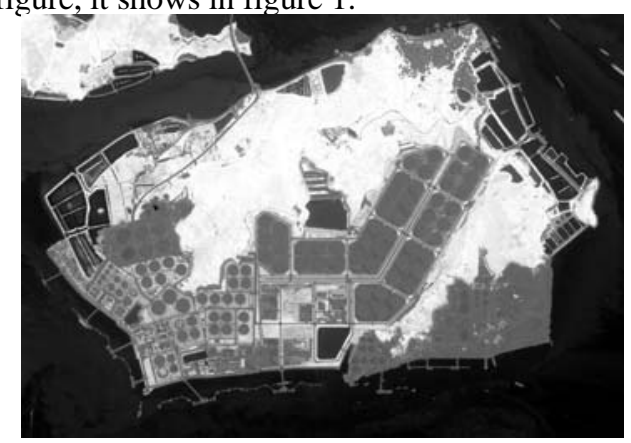

Fig1. NDVI figure

\section{INTERACTIVE STRETCHING EXTRACTING}

The value of Normalized differential vegetation index is less than 1 and bigger than -1 , it shows as $-1 \leqslant$ NDVI $\leqslant 1$. The negative value indicates cloud, water, snow etc; 0 means there are rocks or bare soil etc; the positive value means vegetation coverage.

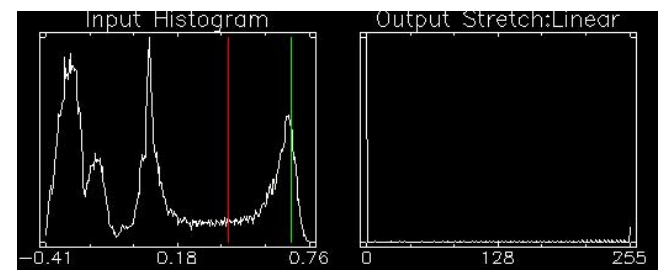

Fig2. NDVI histogram

In this paper the NDVI histogram as shown in figure 2, NDVI value is in 0.41 to 0.76 . In theory the NDVI value greater than 0 is vegetation, but for some noise and error influence, we adopt from the trough value, remove the 
boundary point. As it shows in figure 2, it takes the spectrum between 0.4 and 0.7 to do interactive stretching. After getting the interactive stretching operation, we get the interactive stretching figure, it shows in figure 3.

Though the interactive stretching figure, we know that the vegetation has been fully extracted. But there are some defects that some objects are not adequately separated. Because the affect of multispectral graphics the same spectrum of foreign body, it does not get rid of the breeding zone boundary and the tank farm boundary.

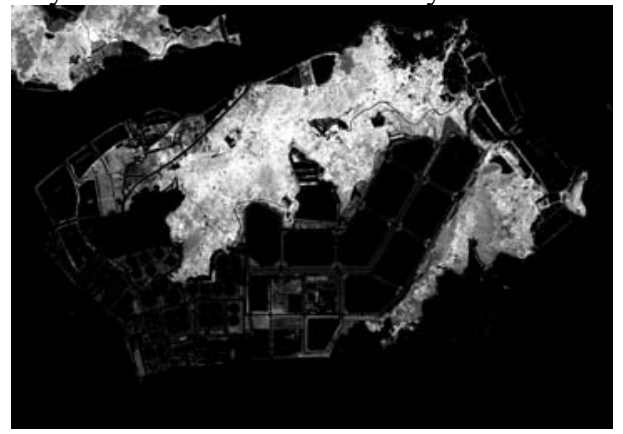

Fig3. Interactive stretching figure

\section{MATHEMATICAL MORPHOLOGY METHOD}

Mathematical morphology makes full use of mathematics and geometry theory ${ }^{[4]}$, in this paper the main use of method is open operation and closed operation of the mathematical morphology. Open operation ${ }^{[5]}$ make the object contour become smooth, it disconnects the narrow gap and eliminates fine prominence. Closed operation is opposite to open operation, but it also makes the contour more smooth, it can remove the narrow gap and long thin gap, eliminate small holes, and fill the contour line of the fracture ${ }^{[6]}$.

In order to extract image for further remove error influence, we use the IDL language to do open operation and closed operation for the interactive stretching figure, the template is circular template, and its size is $5 * 5$. The template is listed below:

$\begin{array}{lllll}0 & 0 & 1 & 0 & 0 \\ 0 & 1 & 1 & 1 & 0 \\ 1 & 1 & 1 & 1 & 1 \\ 0 & 1 & 1 & 1 & 0 \\ 0 & 0 & 1 & 0 & 0\end{array}$

After Open operation and closing operation, vegetation can be extracted adequately from other objects. Our goal is only extracted the Aoshan Island vegetation, so we must get rid of the vegetation outside the island. The method is using Aoshan Island contour graph by mask operation. The mask is a binary image consisting of 0 and 1 . The mask is used in a certain function, when a value is 1 that will be processed, a value of 0 is masked area that will not included in the calculation. The mask formula is using the source image multiply by the binary mask image, it shows in formula(2):

$$
R E S=M A S K * O R I
$$

Where: RES-the result figure after mask operation, MASK-the mask binary image, ORI-the source image.

The contour graph Aoshan Island is based on Normalized Difference Water Index figure using the Count function of
IDL language, the largest area is selected and extracted. By analyzing the characteristics of spectral curve of the water and cartographic background, Normalized Difference Water Index finds out the change rule between them, and it extracts the water with logical discriminant expression. Normalized Difference Water Index(NDWI) is a technology of normalized ratio index based on infrared and near-infrared bands, formula(3):

$$
N D W I=(p(N I R)-p(M I R)) /(p(N I R)+p(M I R))
$$

Where:NIR-near infrared, MIR-intermediate infrared.

According to Normalized Difference Water Index and Count function of IDL, we can get the Aoshan island contour figure,it shows in figure 4

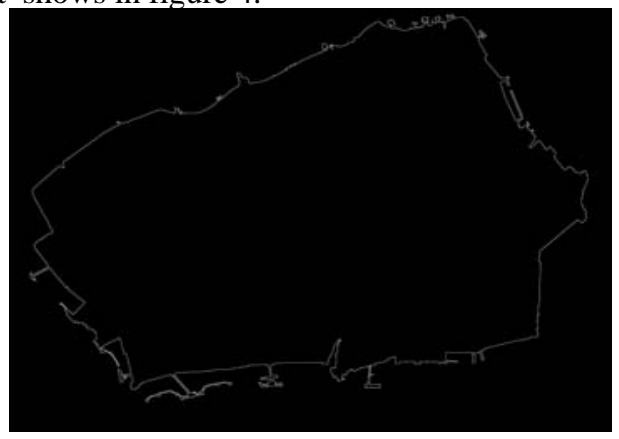

Fig 4. Aoshan island contour figure

After we get the Aoshan island contour figure, we can take the Aoshan island contour figure with Open operation and closing operation image do mask operation. Then we will get the finale result image. The experimental results show in Fig5.

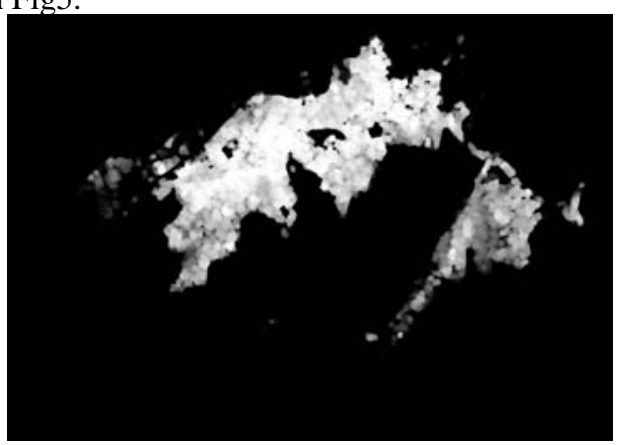

Fig 5. Vegetation extracting figure

\section{CONCLUSION}

For the terrain unique characteristic of Aoshan, we use multispectral remote sensing image normalization vegetation index model, it can actually in a certain range extract vegetation area that we need, but it still not enough precision. And simple mathematical morphology region extraction has greatly error, it can not adapt to the needs of the contemporary. This paper by using IDL language successful combined the normalized vegetation index model with the mathematical morphology of open-closed operation, the vegetation of Aoshan accurately extract from other features, it increased vegetation extraction accuracy. 


\section{ACKNOWLEDGMENT}

Foundation item: Zhejiang province key science and technology special industrial projects(2011C11045)

\section{REFERENCES}

[1] Zhang Zhe,Liu yun-he. Water extraction method based on TM images[J]. Groundwater magazine, 2011,33(5):166-167.

[2] Michael Roderick,Richard Smith,Shane Cridland. The precision of the NDVI derived from AVHRR observations[J].Remote Sensing of Environment,

1996,56(1):57-65.

[3] Alexis Comber, Peter Fisher, Chris Brunsdon, Abdulhakim Khmag.Spatial analysis of remote sensing image classification accuracy[J]. Remote Sensing of Environment,2012,127:237-246.

[4] W.S. Lee, V. Alchanatis, C. Yang, M. Hirafuji, D. Moshou, C. Li. Sensing technologies for precision specialty crop production[J].Computers and Electronics in Agriculture, 2010, 74(1):2-33.

[5] Collin G. Homer, Cameron L. Aldridge, Debra K. Meyer, Spencer J. Schell. Collin G. Homer, Cameron L. Aldridge, Debra K. Meyer, Spencer J. Schell[J]. International Journal of Applied Earth Observation and Geoinformation, 2012, 14(1):233-244.

[6] Fernando Gonzalez. Digital image processing[M]. Beijing:Electronic Industry Press,2007. 